\title{
Strong Summability of Fourier Transforms at Lebesgue Points and Wiener Amalgam Spaces
}

\author{
Ferenc Weisz \\ Department of Numerical Analysis, Eötvös L. University, Pázmány P. Sétány 1/C, Budapest 1117, Hungary \\ Correspondence should be addressed to Ferenc Weisz; weisz@inf.elte.hu
}

Received 1 March 2015; Revised 8 May 2015; Accepted 8 May 2015

Academic Editor: Gelu Popescu

Copyright (C) 2015 Ferenc Weisz. This is an open access article distributed under the Creative Commons Attribution License, which permits unrestricted use, distribution, and reproduction in any medium, provided the original work is properly cited.

We characterize the set of functions for which strong summability holds at each Lebesgue point. More exactly, if $f$ is in the Wiener amalgam space $W\left(L_{1}, \ell_{q}\right)(\mathbb{R})$ and $f$ is almost everywhere locally bounded, or $f \in W\left(L_{p}, \ell_{q}\right)(\mathbb{R})(1<p<\infty, 1 \leq q<\infty)$, then strong $\theta$-summability holds at each Lebesgue point of $f$. The analogous results are given for Fourier series, too.

\section{Introduction}

It was proved by Lebesgue [1] that the Fejér means [2] of the trigonometric Fourier series of an integrable function converge almost everywhere to the function; that is,

$$
\frac{1}{n+1} \sum_{k=0}^{n}\left(s_{k} f(x)-f(x)\right) \longrightarrow 0 \quad \text { as } n \longrightarrow \infty
$$

for almost every $x \in \mathbb{T}$, where $\mathbb{T}$ denotes the torus and $s_{k} f$ the $k$ th partial sum of the Fourier series of the one-dimensional function $f$. The set of convergence is characterized as the Lebesgue points of $f$.

Hardy and Littlewood [3] considered the so-called strong summability and verified that the strong means

$$
\frac{1}{n+1} \sum_{k=0}^{n}\left|s_{k} f(x)-f(x)\right|^{q}
$$

tend to 0 at each Lebesgue point of $f$, as $n \rightarrow \infty$, whenever $f \in L_{p}(\mathbb{T})(1<p<\infty)$ (for Fourier transforms see Giang and Móricz [4]). This result does not hold for $p=1$ (see Hardy and Littlewood [5]). However, the strong means tend to 0 almost everywhere for all $f \in L_{1}(\mathbb{T})$. This is because of Marcinkiewicz [6] for $q=2$ and Zygmund [7] for all $q>0$ (see also Bary [8]). Later Gabisoniya [9] characterized the set of convergence as the so-called Gabisoniya points. Strong summability with lacunary partial sums and Lebesgue points are investigated by Belinsky et al. [10-13].
In a general method of summation, the so-called $\theta$ summation method, which is generated by a single function $\theta$ and which includes the well-known Fejér, Riesz, Weierstrass, and Abel summability methods, is studied intensively in the literature (see, e.g., Butzer and Nessel [14], Trigub and Belinsky [15-17], Liflyand [18], and Weisz [19, 20]). In this paper we generalize some of the above-mentioned results for strong $\theta$-summability of Fourier transforms and for Wiener amalgam spaces. We characterize the set of functions for which strong summability holds at each Lebesgue point. More exactly, we will show that

$$
\lim _{T \rightarrow \infty} \frac{-1}{T} \int_{0}^{\infty} \theta^{\prime}\left(\frac{t}{T}\right)\left|s_{t} f(x)-f(x)\right|^{r} d t=0
$$

at each Lebesgue point $x$ of $f \in W\left(L_{1}, \ell_{q}\right)(\mathbb{R}) \supset L_{q}(\mathbb{R})$ $(1 \leq q<\infty)$ when $f$ is locally bounded at $x$, where $r>0$. Moreover, the convergence holds at each Lebesgue point of $f$ if $f \in W\left(L_{p}, \ell_{q}\right)(\mathbb{R}) \supset L_{p}(\mathbb{R})(1<p<\infty, 1 \leq q<$ $\infty)$. Here $W\left(L_{p}, \ell_{q}\right)(\mathbb{R})$ denotes the Wiener amalgam spaces. Gabisoniya's result was generalized in [21]. The analogous results are given for Fourier series, too.

\section{Wiener Amalgam Spaces and Lebesgue Points}

Let us fix $d \geq 1, d \in \mathbb{N}$. For a set $\mathbb{Y} \neq \emptyset$ let $\mathbb{V}^{d}$ be its Cartesian product $\mathbb{Y} \times \cdots \times \mathbb{Y}$ taken with itself $d$-times. We 
briefly write $L_{p}\left(\mathbb{R}^{d}\right)$ instead of the $L_{p}\left(\mathbb{R}^{d}, \lambda\right)$ space equipped with the norm

$$
\|f\|_{p}:=\left(\int_{\mathbb{R}^{d}}|f(x)|^{p} d \lambda(x)\right)^{1 / p} \quad(1 \leq p<\infty),
$$

with the usual modification for $p=\infty$, where $\lambda$ is the Lebesgue measure. $L_{p}^{\text {loc }}\left(\mathbb{R}^{d}\right)(1 \leq p<\infty)$ denotes the space of measurable functions $f$ for which $|f|^{p}$ is locally integrable. We say that $f$ is locally bounded at $x$ if there exists a neighborhood of $x$ such that $f$ is bounded on this neighborhood.

Now we generalize the $L_{p}$ spaces. A measurable function $f$ belongs to the Wiener amalgam space $W\left(L_{p}, \ell_{q}\right)\left(\mathbb{R}^{d}\right)(1 \leq$ $p, q \leq \infty)$ if

$$
\|f\|_{W\left(L_{p}, \ell_{q}\right)}:=\left(\sum_{k \in \mathbb{Z}^{d}}\|f(\cdot+k)\|_{L_{p}[0,1)^{d}}^{q}\right)^{1 / q}<\infty,
$$

with the obvious modification for $q=\infty$. It is easy to see that $W\left(L_{p}, \ell_{p}\right)\left(\mathbb{R}^{d}\right)=L_{p}\left(\mathbb{R}^{d}\right)$ and the following continuous embeddings hold true:

$$
\begin{array}{ll}
W\left(L_{p_{1}}, \ell_{q}\right)\left(\mathbb{R}^{d}\right) \supset W\left(L_{p_{2}}, \ell_{q}\right)\left(\mathbb{R}^{d}\right) & \left(p_{1} \leq p_{2}\right), \\
W\left(L_{p}, \ell_{q_{1}}\right)\left(\mathbb{R}^{d}\right) \subset W\left(L_{p}, \ell_{q_{2}}\right)\left(\mathbb{R}^{d}\right) & \left(q_{1} \leq q_{2}\right),
\end{array}
$$

$\left(1 \leq p_{1}, p_{2}, q_{1}, q_{2} \leq \infty\right)$. Thus

$$
\begin{array}{r}
W\left(L_{\infty}, \ell_{1}\right)\left(\mathbb{R}^{d}\right) \subset L_{p}\left(\mathbb{R}^{d}\right) \subset W\left(L_{1}, \ell_{\infty}\right)\left(\mathbb{R}^{d}\right) \\
(1 \leq p \leq \infty) .
\end{array}
$$

A point $x \in \mathbb{R}^{d}$ is called a $p$-Lebesgue point (or a Lebesgue point of order $p$ ) of $f \in L_{p}^{\text {loc }}\left(\mathbb{R}^{d}\right)$ if

$$
\begin{aligned}
& \lim _{h \rightarrow 0}\left(\frac{1}{(2 h)^{d}} \int_{-h}^{h} \cdots \int_{-h}^{h}|f(x-s)-f(x)|^{p} d s\right)^{1 / p} \\
& \quad=0 .
\end{aligned}
$$

It was proved by Feichtinger and Weisz $[22,23]$ that almost every point $x \in \mathbb{R}^{d}$ is a $p$-Lebesgue point of $f \in$ $W\left(L_{p}, \ell_{\infty}\right)\left(\mathbb{R}^{d}\right)(1 \leq p<\infty)$. In context of Lebesgue points of $L_{p}$-functions we call also for the earlier papers of Belinsky et al. $[12,13]$.

In this paper the constants $C$ and $C_{p}$ may vary from line to line and the constants $C_{p}$ are depending only on $p$.

\section{The Kernel Functions}

The Fourier transform of $f \in L_{1}(\mathbb{R})$ is given by

$$
\widehat{f}(x)=\frac{1}{(2 \pi)^{1 / 2}} \int_{\mathbb{R}} f(u) e^{-\imath x u} d u \quad(x \in \mathbb{R}),
$$

where $\imath=\sqrt{-1}$. Suppose first that $f \in L_{p}(\mathbb{R})$ for some $1 \leq$ $p \leq 2$. The Fourier inversion formula

$$
f(x)=\frac{1}{(2 \pi)^{1 / 2}} \int_{\mathbb{R}} \widehat{f}(u) e^{i x u} d u
$$

$$
\left(x \in \mathbb{R}, \widehat{f} \in L_{1}(\mathbb{R})\right)
$$

motivates the definition of the Dirichlet integral $s_{t} f(t>0)$ introduced by

$$
\begin{aligned}
s_{t} f(x) & :=\frac{1}{(2 \pi)^{1 / 2}} \int_{-t}^{t} \widehat{f}(u) e^{\imath x u} d u \\
& =\frac{1}{2 \pi} \int_{\mathbb{R}} f(x-u) D_{t}(u) d u,
\end{aligned}
$$

where the Dirichlet kernel is defined by

$$
D_{t}(x):=\int_{-t}^{t} e^{\imath x u} d u=2 \frac{\sin (t x)}{x} .
$$

Obviously, $\left|D_{t}\right| \leq C t$.

It is easy to see that, with the help of the integral in (11), the definition of $s_{t} f$ can be extended to all $f \in W\left(L_{1}, \ell_{q}\right)(\mathbb{R})$ with $1 \leq q<\infty$. Note that $W\left(L_{1}, \ell_{p}\right)(\mathbb{R}) \supset L_{p}(\mathbb{R})$, where $1 \leq p<\infty$. It is known (see, e.g., Grafakos [24] or [20]) that, for $f \in L_{p}(\mathbb{R}), 1<p<\infty$,

$$
\lim _{T \rightarrow \infty} s_{T} f=f \quad \text { in the } L_{p}(R) \text {-norm and a.e. }
$$

This convergence does not hold for $p=1$. However, using a summability method, we can generalize these results. We may take a general summability method, the so-called $\theta$-summation defined by a function $\theta: \mathbb{R}_{+} \rightarrow \mathbb{R}$. This summation contains all well-known summability methods, such as the Marcinkiewicz-Fejér, Riesz, Weierstrass, Abel, Picard, and Bessel summations.

Suppose that $\theta$ is continuous on $\mathbb{R}_{+}$; the support of $\theta$ is $[0, c]$ for some $0<c \leq \infty$ and $\theta$ is differentiable on $(0, c)$. Suppose further that

$$
\begin{aligned}
\theta(0) & =1, \\
\int_{0}^{\infty}(t \vee 1)^{d}\left|\theta^{\prime}(t)\right| d t & <\infty, \\
\lim _{t \rightarrow \infty} t^{d} \theta(t) & =0,
\end{aligned}
$$

where $d \in \mathbb{N}, \vee$ denotes the maximum, and $\wedge$ denotes the minimum.

For $T>0$ the $\theta$-means of a function $f \in L_{p}(\mathbb{R})(1 \leq p \leq$ 2) are defined by

$$
\sigma_{T}^{\theta} f(x):=\frac{1}{(2 \pi)^{1 / 2}} \int_{\mathbb{R}} \theta\left(\frac{|u|}{T}\right) \hat{f}(u) e^{\imath x u} d u .
$$

It is easy to see that

$$
\sigma_{T}^{\theta} f(x)=\frac{1}{(2 \pi)^{1 / 2}} \int_{\mathbb{R}} f(x-u) K_{T}^{\theta}(u) d u .
$$


Note that this formula is well defined for all $f \in$ $W\left(L_{1}, \ell_{\infty}\right)(\mathbb{R})$. Here

$$
\begin{aligned}
K_{T}^{\theta}(x) & :=\frac{1}{(2 \pi)^{1 / 2}} \int_{\mathbb{R}} \theta\left(\frac{|u|}{T}\right) e^{\imath x u} d u \\
& =\frac{-1}{(2 \pi)^{1 / 2} T} \int_{\mathbb{R}} \int_{|u|}^{\infty} \theta^{\prime}\left(\frac{t}{T}\right) d t e^{\imath x u} d u \\
& =\frac{-1}{(2 \pi)^{1 / 2} T} \int_{0}^{\infty} \theta^{\prime}\left(\frac{t}{T}\right) \int_{-t}^{t} e^{\imath x u} d u d t \\
& =\frac{-1}{(2 \pi)^{1 / 2} T} \int_{0}^{\infty} \theta^{\prime}\left(\frac{t}{T}\right) D_{t}(x) d t
\end{aligned}
$$

denotes the $\theta$-kernel. Thus

$$
\sigma_{T}^{\theta} f(x)=\frac{-1}{T} \int_{0}^{\infty} \theta^{\prime}\left(\frac{t}{T}\right) s_{t} f(x) d t
$$

for all $f \in W\left(L_{1}, \ell_{q}\right)(\mathbb{R})$ with $1 \leq q<\infty$. Note that for the Fejér means (i.e., for $\theta(t)=\max ((1-|t|), 0))$ we get the usual definition

$$
\sigma_{T}^{\theta} f(x)=\frac{1}{T} \int_{0}^{T} s_{t} f(x) d t
$$

In Feichtinger and Weisz [22, 23] we have proved that, under conditions (14) and (25) with $d=1$,

$$
\lim _{T \rightarrow \infty} \sigma_{T}^{\theta} f(x)=f(x)
$$

for all Lebesgue points of $f \in W\left(L_{1}, \ell_{\infty}\right)(\mathbb{R})$. In this paper, we investigate the problem of the strong summability, that is, whether the convergence

$$
\lim _{T \rightarrow \infty} \frac{-1}{T} \int_{0}^{\infty} \theta^{\prime}\left(\frac{t}{T}\right)\left|s_{t} f(x)-f(x)\right|^{r} d t=0
$$

holds for Lebesgue points and some $r>0$. Usually $\theta$ is increasing; then we can take the absolute value of $\theta^{\prime}$ in the integral.

To this end we have to introduce some $d$-dimensional definitions. In the $d$-dimensional case we define the Dirichlet kernel by

$$
D_{t}(x):=\prod_{i=1}^{d} D_{t}\left(x_{i}\right)=2^{d} \prod_{i=1}^{d} \frac{\sin \left(t x_{i}\right)}{x_{i}} \quad(t>0)
$$

and the so called Marcinkiewicz- $\theta$-kernel by

$$
K_{T}^{\theta}(x)=\frac{-1}{(2 \pi)^{d / 2} T} \int_{0}^{\infty} \theta^{\prime}\left(\frac{t}{T}\right) D_{t}(x) d t \quad(T>0),
$$

where $x=\left(x_{1}, \ldots, x_{d}\right) \in \mathbb{R}^{d}$. In [21], we have seen that we may suppose that $x_{1}>x_{2}>\cdots>x_{d}>0$ and $x_{1}-\sum_{j=2}^{d} x_{j}>0$ and we proved the next lemma. Denote by

$$
\operatorname{soc} t:= \begin{cases}\cos t, & \text { if } d \text { is even; } \\ \sin t, & \text { if } d \text { is odd }\end{cases}
$$

Lemma 1. Let

$$
\begin{aligned}
\left|\int_{0}^{\infty} \theta^{\prime}(t) t^{i}(s o c)^{(i)}(t u) d t\right| \leq C u^{-\alpha} & \\
& (i=0, \ldots, d-1)
\end{aligned}
$$

for some $0<\alpha<\infty$. Then

$$
\left|K_{T}^{\theta}(x)\right| \leq C T^{d-j} x_{1}^{-1} \cdots x_{j}^{-1} \quad(j=0, \ldots, d) .
$$

If in addition $x_{1}-\sum_{j=2}^{d} x_{j}>1 / T$ and $x_{j+1}<1 / T$, where $x_{d+1}=$ 0 , then

$$
\begin{aligned}
\left|K_{T}^{\theta}(x)\right| \leq C T^{-\alpha+d-j} x_{1}^{-1} \cdots x_{j}^{-1}\left(x_{1}-\sum_{j=2}^{d} x_{j}\right)^{-\alpha} & \\
& (j=1, \ldots, d) .
\end{aligned}
$$

The next lemma is due to the author [25].

Lemma 2. If (14) and (25) are satisfied for some $d \in \mathbb{N}$ and $0<\alpha<\infty$, then $\int_{\mathbb{R}^{d}}\left|K_{T}^{\theta}\right| d \lambda \leq C\left(T \in \mathbb{R}_{+}\right)$.

\section{Strong Summability of Fourier Transforms}

In this section we characterize a wide set of functions for which strong summability holds at each Lebesgue point. For the convergence of $f \in W\left(L_{p}, \ell_{q}\right)(\mathbb{R})(1<p<\infty, 1 \leq q<$ $\infty)$ at $p$-Lebesgue points we proved the following result in [21]. Note that $W\left(L_{p}, \ell_{p}\right)(\mathbb{R})=L_{p}(\mathbb{R})$.

Theorem 3. Suppose that (14) and (25) hold for some $d \in \mathbb{N}$ and $0<\alpha<\infty$. Let $f \in W\left(L_{p}, \ell_{q}\right)(\mathbb{R})$ for some $1<p<\infty$ and $1 \leq q<\infty$. If $x_{j}$ is a $p$-Lebesgue point of $f$ for all $j=$ $1, \ldots, d$, then

$$
\lim _{T \rightarrow \infty} \frac{-1}{T} \int_{0}^{\infty} \theta^{\prime}\left(\frac{t}{T}\right) \prod_{j=1}^{d}\left(s_{t} f\left(x_{j}\right)-f\left(x_{j}\right)\right) d t=0 .
$$

If all $x_{j}(j=1, \ldots, d)$ are equal, then we obtain the following.

Corollary 4. Suppose that (14) and (25) hold for some even $d \in \mathbb{N}$ and $0<\alpha<\infty$. Let $f \in W\left(L_{p}, \ell_{q}\right)(\mathbb{R})$ for some $1<p<\infty$ and $1 \leq q<\infty$. If $x \in \mathbb{R}$ is a $p$-Lebesgue point of $f$, then

$$
\lim _{T \rightarrow \infty} \frac{-1}{T} \int_{0}^{\infty} \theta^{\prime}\left(\frac{t}{T}\right)\left|s_{t} f(x)-f(x)\right|^{d} d t=0 .
$$

Obviously, the convergence holds almost everywhere. Corollary 4 does not hold for $p=1$ (see Hardy and Littlewood [5]). However, we [21] extended it for $p=1$, but for much more specialized points than the Lebesgue points, for the so-called Gabisoniya points, which were introduced in [9]. In the next theorem we generalize Theorem 3 and Corollary 4 for $p=1$ and for a subspace of $W\left(L_{1}, \ell_{q}\right)(\mathbb{R})$. 
Theorem 5. Suppose that (14) and (25) hold for some $d \in \mathbb{N}$ and $0<\alpha<\infty$. Let $f \in W\left(L_{1}, \ell_{q}\right)(\mathbb{R})$ for some $1 \leq q<\infty$. If $x_{j}$ is a Lebesgue point of $f$ and $f$ is locally bounded at $x_{j}$ for all $j=1, \ldots, d$, then

$$
\lim _{T \rightarrow \infty} \frac{-1}{T} \int_{0}^{\infty} \theta^{\prime}\left(\frac{t}{T}\right) \prod_{j=1}^{d}\left(s_{t} f\left(x_{j}\right)-f\left(x_{j}\right)\right) d t=0
$$

Proof. It is easy to see that

$$
\begin{gathered}
\frac{-1}{T} \int_{0}^{\infty} \theta^{\prime}\left(\frac{t}{T}\right) \prod_{j=1}^{d}\left(s_{t} f\left(x_{j}\right)-f\left(x_{j}\right)\right) d t=\frac{-1}{T} \\
\cdot \int_{0}^{\infty} \theta^{\prime}\left(\frac{t}{T}\right) \prod_{j=1}^{d}\left(\frac{1}{2 \pi} \int_{\mathbb{R}} f\left(x_{j}-s_{j}\right) D_{t}\left(s_{j}\right) d s_{j}\right. \\
\left.-f\left(x_{j}\right)\right) d t=\frac{-1}{T} \int_{0}^{\infty} \theta^{\prime}\left(\frac{t}{T}\right) \prod_{j=1}^{d} \lim _{n \rightarrow \infty} \frac{1}{2 \pi} \\
\cdot \int_{-n}^{n}\left(f\left(x_{j}-s_{j}\right)-f\left(x_{j}\right)\right) D_{t}\left(s_{j}\right) d s_{j} d t .
\end{gathered}
$$

Since

$$
\begin{aligned}
& \left|\int_{-n}^{n} f\left(x_{j}-s_{j}\right) D_{t}\left(s_{j}\right) d s_{j}\right| \leq C t\|f\|_{W\left(L_{1}, \ell_{q}\right)}, \\
& \left|\int_{-n}^{n} f\left(x_{j}\right) D_{t}\left(s_{j}\right) d s_{j}\right|=\left|f\left(x_{j}\right)\right|\left|\int_{-n}^{n} \frac{\sin \left(t s_{j}\right)}{s_{j}} d s_{j}\right| \\
& \quad=\left|f\left(x_{j}\right)\right|\left|\int_{-n t}^{n t} \frac{\sin u}{u} d u\right| \leq C\left|f\left(x_{j}\right)\right|,
\end{aligned}
$$

we obtain

$$
\begin{aligned}
& \frac{-1}{T} \int_{0}^{\infty} \theta^{\prime}\left(\frac{t}{T}\right) \prod_{j=1}^{d}\left(s_{t} f\left(x_{j}\right)-f\left(x_{j}\right)\right) d t=\lim _{n \rightarrow \infty} \frac{-1}{T} \\
& \cdot \int_{0}^{\infty} \theta^{\prime}\left(\frac{t}{T}\right) \\
& \cdot \frac{1}{(2 \pi)^{d}} \int_{[-n, n]^{d}} \prod_{j=1}^{d}\left(f\left(x_{j}-s_{j}\right)-f\left(x_{j}\right)\right) \\
& \cdot D_{t}(s) d s d t=\lim _{n \rightarrow \infty} \frac{1}{(2 \pi)^{d}} \\
& \cdot \int_{[-n, n]^{d}} \frac{-1}{T} \int_{0}^{\infty} \theta^{\prime}\left(\frac{t}{T}\right) \prod_{j=1}^{d}\left(f\left(x_{j}-s_{j}\right)-f\left(x_{j}\right)\right) \\
& \cdot D_{t}(s) d t d s=\frac{1}{(2 \pi)^{d / 2}} \int_{\mathbb{R}^{d}} \prod_{j=1}^{d}\left(f\left(x_{j}-s_{j}\right)\right. \\
& \left.\quad-f\left(x_{j}\right)\right) K_{T}^{\theta}(s) d s .
\end{aligned}
$$

For simplicity we will prove the rest of the theorem for $d=3$, only. It can be proved for higher dimensions similarly. As we mentioned earlier, we may suppose that $s_{1}>s_{2}>s_{3}>$ 0 and $s_{1}-s_{2}-s_{3}>0$. Let us fix a small $r>0$ and denote the square $[0, r / 2]^{3}$ by $S_{r / 2}$. We can prove in the same way as we did in Theorem 4 of [21] that

$$
\int_{S_{r / 2}} \prod_{j=1}^{3}\left|f\left(x_{j}-s_{j}\right)-f\left(x_{j}\right)\right|\left|K_{T}^{\theta}(s)\right| d s<\epsilon
$$

if $r$ is small enough and $T$ is large enough. The estimation of this integral on the set $S_{r / 2}^{c}$ of that proof does not work now, because there we used a modified maximal function which is not necessarily bounded in our case. So we have to show here that

$$
\lim _{T \rightarrow \infty} \int_{S_{r / 2}^{c}} \prod_{j=1}^{3}\left|f\left(x_{j}-s_{j}\right)-f\left(x_{j}\right)\right|\left|K_{T}^{\theta}(s)\right| d s=0 .
$$

To this end let us introduce the sets

$$
\begin{aligned}
& A_{1}:=\left\{s: s_{1}>\frac{r}{2}, 0<s_{3}<s_{2}<\frac{1}{T}\right\}, \\
& A_{2}:=\left\{s: s_{1}>\frac{r}{2}, 0<s_{3}<\frac{1}{T}<s_{2}<\delta\right\}, \\
& A_{3}:=\left\{s: s_{1}>\frac{r}{2}, \frac{1}{T}<s_{3}<s_{2}<\delta\right\}, \\
& A_{4}:=\left\{s: s_{1}>\frac{r}{2}, 0<s_{3}<\frac{1}{T}<\delta<s_{2}\right\}, \\
& A_{5}:=\left\{s: s_{1}>\frac{r}{2}, \frac{1}{T}<s_{3}<\delta<s_{2}\right\}, \\
& A_{6}:=\left\{s: s_{1}>\frac{r}{2}, \frac{1}{T}<\delta<s_{3}<s_{2}\right\}, \\
& B_{1}:=\left\{s: 0<s_{1}-s_{2}-s_{3}<\frac{1}{T}\right\}, \\
& B_{2}:=\left\{s: \frac{1}{T}<s_{1}-s_{2}-s_{3}<\delta\right\}, \\
& B_{3}:=\left\{s: \delta<s_{1}-s_{2}-s_{3}<\frac{\left(s_{1}-s_{2}\right)}{2}\right\},
\end{aligned}
$$

$B_{4}$

$$
:=\left\{s: \frac{\left(s_{1}-s_{2}\right)}{2} \vee \delta<s_{1}-s_{2}-s_{3}<s_{1}-s_{2}-\delta\right\},
$$$$
B_{5}:=\left\{s:\left(s_{1}-s_{2}-\delta\right) \vee \delta<s_{1}-s_{2}-s_{3}<s_{1}-s_{2}\right\} \text {, }
$$$$
C_{1}:=\left\{s: 0<s_{1}-s_{2}<\delta\right\} \text {, }
$$$$
C_{2}:=\left\{s: \delta<s_{1}-s_{2}<\frac{s_{1}}{2}\right\},
$$$$
C_{3}:=\left\{s: \frac{s_{1}}{2}<s_{1}-s_{2}<s_{1}-\delta\right\},
$$

$C_{4}:=\left\{s: s_{1}-\delta<s_{1}-s_{2}<s_{1}\right\}$ 
for a given small $\delta>0$ and large $T$. Then we have to estimate the integral in (35) for $A_{i}, i=1, \ldots, 6$. First of all observe that $A_{i} \cap B_{j}=\emptyset$ for $i=1,2,3$ and $j=1,2$. On the set $A_{1}$ we have $s_{2}, s_{3}<1 / T$ and so $s_{1}-s_{2}-s_{3}>s_{1} / 2$. Hence

$$
\left|K_{T}^{\theta}(s)\right| \leq C T^{2-\alpha} s_{1}^{-1}\left(s_{1}-s_{2}-s_{3}\right)^{-\alpha} \leq C T^{2-\alpha} s_{1}^{-1-\alpha} .
$$

Since $f$ is locally bounded at $x_{j}$, we get by (37) that

$$
\begin{gathered}
\int_{A_{1} \cap\left(B_{3} \cup B_{4} \cup B_{5}\right)} \prod_{j=1}^{3}\left|f\left(x_{j}-s_{j}\right)-f\left(x_{j}\right)\right|\left|K_{T}^{\theta}(s)\right| d s \\
\leq C T^{2-\alpha} \int_{r / 2}^{\infty} \int_{0}^{1 / T} \int_{0}^{1 / T} s_{1}^{-1-\alpha} \prod_{j=1}^{3}\left|f\left(x_{j}-s_{j}\right)\right| d s \\
\leq C T^{-\alpha} \sum_{i=0}^{\infty}(i \vee 1)^{-1-\alpha} \int_{i}^{i+1}\left|f\left(x_{1}-s_{1}\right)\right| d s_{1} \\
\leq C T^{-\alpha}\|f\|_{W\left(L_{1}, \ell_{\infty}\right)} \longrightarrow 0,
\end{gathered}
$$

as $T \rightarrow \infty$. On $A_{2}$ we have

$$
\begin{aligned}
\left|K_{T}^{\theta}(s)\right| & \leq C T^{1-\alpha} s_{1}^{-1} s_{2}^{-1}\left(s_{1}-s_{2}-s_{3}\right)^{-\alpha} \\
& \leq C T^{1-\alpha} s_{1}^{-1-\alpha} s_{2}^{-1}
\end{aligned}
$$

and so

$$
\begin{aligned}
& \int_{A_{2} \cap\left(B_{3} \cup B_{4} \cup B_{5}\right)} \prod_{j=1}^{3}\left|f\left(x_{j}-s_{j}\right)-f\left(x_{j}\right)\right|\left|K_{T}^{\theta}(s)\right| d s \\
& \leq C T^{1-\alpha} \int_{r / 2}^{\infty} \int_{1 / T}^{\delta} \int_{0}^{1 / T} s_{1}^{-1-\alpha} s_{2}^{-1} \prod_{j=1}^{3}\left|f\left(x_{j}-s_{j}\right)\right| d s \\
& \leq C T^{-\alpha} \ln T \sum_{i=0}^{\infty}(i \vee 1)^{-1-\alpha} \int_{i}^{i+1}\left|f\left(x_{1}-s_{1}\right)\right| d s_{1} \\
& \leq C T^{-\alpha} \ln T\|f\|_{W\left(L_{1}, \ell_{\infty}\right)} \longrightarrow 0 .
\end{aligned}
$$

Similarly,

$$
\begin{aligned}
& \int_{A_{3} \cap\left(B_{3} \cup B_{4} \cup B_{5}\right)} \prod_{j=1}^{3}\left|f\left(x_{j}-s_{j}\right)-f\left(x_{j}\right)\right|\left|K_{T}^{\theta}(s)\right| d s \\
& \leq C T^{-\alpha} \int_{r / 2}^{\infty} \int_{1 / T}^{\delta} \int_{1 / T}^{\delta} s_{1}^{-1-\alpha} s_{2}^{-1} s_{3}^{-1} \prod_{j=1}^{3}\left|f\left(x_{j}-s_{j}\right)\right| d s \\
& \leq C T^{-\alpha} \ln ^{2} T\|f\|_{W\left(L_{1}, \ell_{\infty}\right)} \longrightarrow 0 .
\end{aligned}
$$

On the set $A_{4} \cap B_{1}$ we have $s_{1}-s_{2}<2 / T<\delta$ if $T$ is large enough and so $s_{2}>s_{1} / 2$. Then

$$
\begin{aligned}
& \left|K_{T}^{\theta}(s)\right| \leq C T s_{1}^{-1} s_{2}^{-1} \leq C T s_{1}^{-2}, \\
& \int_{A_{4} \cap B_{1}} \prod_{j=1}^{3}\left|f\left(x_{j}-s_{j}\right)-f\left(x_{j}\right)\right|\left|K_{T}^{\theta}(s)\right| d s \\
& \leq C T \int_{r / 2}^{\infty} \int_{s_{1}-\delta}^{s_{1}} \int_{0}^{1 / T} s_{1}^{-2} \prod_{j=1}^{3}\left|f\left(x_{j}-s_{j}\right)\right| d s \\
& \leq C \sum_{i=0}^{N_{0}-1}(i \vee 1)^{-2} \\
& \cdot \int_{i}^{i+1} \int_{s_{1}-\delta}^{s_{1}}\left|f\left(x_{1}-s_{1}\right)\right|\left|f\left(x_{2}-s_{2}\right)\right| d s_{1} d s_{2} \\
& +C \sum_{i=N_{0}}^{\infty} i^{-2} \\
& \quad \cdot \int_{i}^{i+1} \int_{s_{1}-\delta}^{s_{1}}\left|f\left(x_{1}-s_{1}\right)\right|\left|f\left(x_{2}-s_{2}\right)\right| d s_{1} d s_{2} \\
& \leq C\left\|f \times f 1_{\left\{\left(s_{1}, s_{2}\right): 0<s_{1}<N_{0}, s_{1}-\delta<s_{2} \leq s_{1}\right\}}\right\|_{W\left(L_{1}, \ell_{\infty}\right)} \\
& \quad+C N_{0}^{-1}\|f \times f\|_{W\left(L_{1}, \ell_{\infty}\right)} .
\end{aligned}
$$

The second term is less than $\epsilon$ if $N_{0}$ is large enough and the first term is less than $\epsilon$ if $\delta$ is small enough. The set $A_{4} \cap B_{2}$ can be handled in the same way.

On $A_{4} \cap\left(B_{3} \cup B_{4} \cup B_{5}\right)$ we have $s_{1}-s_{2}>\delta$. Thus $\left(B_{3} \cup\right.$ $\left.B_{4} \cup B_{5}\right) \cap C_{1}=\emptyset$ and $s_{1}-s_{2}-s_{3}>s_{1}-s_{2}-1 / T>\left(s_{1}-s_{2}\right) / 2$. Then

$$
\begin{aligned}
\left|K_{T}^{\theta}(s)\right| & \leq C T^{1-\alpha} s_{1}^{-1} s_{2}^{-1}\left(s_{1}-s_{2}-s_{3}\right)^{-\alpha} \\
& \leq C T^{1-\alpha} s_{1}^{-1} s_{2}^{-1}\left(s_{1}-s_{2}\right)^{-\alpha} .
\end{aligned}
$$

On $C_{2}$ we have

$$
\begin{aligned}
\left|K_{T}^{\theta}(s)\right| & \leq C T^{1-\alpha} s_{1}^{-2}\left(s_{1}-s_{2}\right)^{-\alpha} \\
& \leq C T^{1-\alpha} s_{1}^{-1-\eta}\left(s_{1}-s_{2}\right)^{-1-\alpha+\eta},
\end{aligned}
$$

where $\eta$ is chosen such that $0<\eta<1 \wedge \alpha$. Hence

$$
\begin{aligned}
& \int_{A_{4} \cap\left(B_{3} \cup B_{4} \cup B_{5}\right) \cap C_{2}} \prod_{j=1}^{3}\left|f\left(x_{j}-s_{j}\right)-f\left(x_{j}\right)\right|\left|K_{T}^{\theta}(s)\right| d s \\
& \leq C T^{1-\alpha} \int_{r / 2}^{\infty} \int_{s_{1} / 2}^{s_{1}-\delta} \int_{0}^{1 / T} s_{1}^{-1-\eta}\left(s_{1}-s_{2}\right)^{-1-\alpha+\eta} \\
& \cdot \prod_{j=1}^{3}\left|f\left(x_{j}-s_{j}\right)\right| d s \leq C T^{-\alpha} \sum_{i=0}^{\infty}(i \vee 1)^{-1-\eta} \\
& \cdot \sum_{0 \leq j<(i+1) / 2}(j \vee 1)^{-1-\alpha+\eta} \cdot \int_{i}^{i+1} \int_{s_{1}-j-1}^{s_{1}-j}\left|f\left(x_{1}-s_{1}\right)\right| \\
& \cdot\left|f\left(x_{2}-s_{2}\right)\right| d s_{1} d s_{2} \leq C T^{-\alpha}\|f\|_{W\left(L_{1}, \ell_{\infty}\right)}^{2} \\
& \longrightarrow 0 .
\end{aligned}
$$


On $C_{3}$, (44) implies that

$$
\begin{aligned}
& \left|K_{T}^{\theta}(s)\right| \leq C T^{1-\alpha} s_{1}^{-1-\alpha} s_{2}^{-1} \leq C T^{1-\alpha} s_{1}^{-1-\alpha+\eta} s_{2}^{-1-\eta}, \\
& \int_{A_{4} \cap\left(B_{3} \cup B_{4} \cup B_{5}\right) \cap C_{3}} \prod_{j=1}^{3}\left|f\left(x_{j}-s_{j}\right)-f\left(x_{j}\right)\right|\left|K_{T}^{\theta}(s)\right| d s \\
& \leq C T^{1-\alpha} \int_{r / 2}^{\infty} \int_{\delta}^{s_{1} / 2} \int_{0}^{1 / T} s_{1}^{-1-\alpha+\eta} s_{2}^{-1-\eta} \prod_{j=1}^{3}\left|f\left(x_{j}-s_{j}\right)\right| d s \\
& \leq C T^{-\alpha} \sum_{i=0}^{\infty}(i \vee 1)^{-1-\alpha+\eta} \sum_{0 \leq j<(i+1) / 2}(j \vee 1)^{-1-\eta} \\
& \cdot \int_{i}^{i+1} \int_{j}^{j+1}\left|f\left(x_{1}-s_{1}\right)\right|\left|f\left(x_{2}-s_{2}\right)\right| d s_{1} d s_{2} \\
& \leq C T^{-\alpha}\|f\|_{W\left(L_{1}, \ell_{\infty}\right)}^{2} \longrightarrow 0 .
\end{aligned}
$$

Observe that $s_{2}>\delta$ contradicts $C_{4}$.

Consider the set $A_{5} \cap B_{1}$. If $s_{1}-s_{2}>2 / T$, then $s_{3}>$ $s_{1}-s_{2}-1 / T>\left(s_{1}-s_{2}\right) / 2$ and if $s_{1}-s_{2}<2 / T$, then $s_{3}>1 / T>$ $\left(s_{1}-s_{2}\right) / 2$. Observe that $s_{1}-s_{2}<1 / T+\delta<2 \delta$. Hence

$$
\left|K_{T}^{\theta}(s)\right| \leq C s_{1}^{-1} s_{2}^{-1} s_{3}^{-1} \leq C s_{1}^{-2}\left(s_{1}-s_{2}\right)^{-1 / 2} s_{3}^{-1 / 2} .
$$

Since $s_{3}<\delta$, we can integrate in $s_{3}$ to obtain

$$
\begin{aligned}
& \int_{A_{5} \cap B_{1}} \prod_{j=1}^{3}\left|f\left(x_{j}-s_{j}\right)-f\left(x_{j}\right)\right|\left|K_{T}^{\theta}(s)\right| d s \\
& \leq C \int_{r / 2}^{\infty} \int_{s_{1}-2 \delta}^{s_{1}} \int_{s_{1}-s_{2}-1 / T}^{s_{1}-s_{2}} s_{1}^{-2}\left(s_{1}-s_{2}\right)^{-1 / 2} \\
& \cdot s_{3}^{-1 / 2} \prod_{j=1}^{3}\left|f\left(x_{j}-s_{j}\right)\right| d s \\
& \leq C \int_{r / 2}^{\infty} \int_{s_{1}-2 \delta}^{s_{1}} s_{1}^{-2}\left|f\left(x_{1}-s_{1}\right)\right|\left|f\left(x_{2}-s_{2}\right)\right| d s_{1} d s_{2} \\
& \leq C\left\|f \times f 1_{\left\{\left(s_{1}, s_{2}\right): 0<s_{1}<N_{0}, s_{1}-2 \delta<s_{2} \leq s_{1}\right\}}\right\|_{W\left(L_{1}, \ell_{\infty}\right)} \\
& +C N_{0}^{-1}\|f \times f\|_{W\left(L_{1}, \ell_{\infty}\right)}
\end{aligned}
$$

as in (43). Similarly, $s_{1}-s_{2}<2 \delta$ holds as well on $A_{5} \cap B_{2}$. If $s_{1}-s_{2}-s_{3}>s_{3}$, then

$$
\begin{aligned}
\left|K_{T}^{\theta}(s)\right| & \leq C T^{-\alpha} s_{1}^{-1} s_{2}^{-1} s_{3}^{-1}\left(s_{1}-s_{2}-s_{3}\right)^{-\alpha} \\
& \leq C T^{-\alpha} s_{1}^{-2} s_{3}^{-1-\alpha}
\end{aligned}
$$

and so

$$
\begin{aligned}
& \int_{A_{5} \cap B_{2}} \prod_{j=1}^{3}\left|f\left(x_{j}-s_{j}\right)-f\left(x_{j}\right)\right|\left|K_{T}^{\theta}(s)\right| d s \\
& \leq C T^{-\alpha} \int_{r / 2}^{\infty} \int_{s_{1}-2 \delta}^{s_{1}} \int_{1 / T}^{\delta} s_{1}^{-2} s_{3}^{-1-\alpha} \prod_{j=1}^{3}\left|f\left(x_{j}-s_{j}\right)\right| d s \\
& \leq C \int_{r / 2}^{\infty} \int_{s_{1}-2 \delta}^{s_{1}} s_{1}^{-2}\left|f\left(x_{1}-s_{1}\right)\right|\left|f\left(x_{2}-s_{2}\right)\right| d s_{1} d s_{2} \\
& <\epsilon
\end{aligned}
$$

Moreover, $B_{5}^{\prime}$ contradicts $A_{5}$, more exactly, $s_{3}>1 / T$.

On $A_{6} s_{3}>\delta$ and $s_{1}-s_{2}-s_{3}>0$, thus $s_{1}-s_{2}>\delta$. Similar to $(48)$,

$$
\left|K_{T}^{\theta}(s)\right| \leq C s_{1}^{-1} s_{2}^{-1} s_{3}^{-1} \leq C s_{1}^{-2}\left(s_{1}-s_{2}\right)^{-1}
$$


on $A_{6} \cap B_{1} \cap C_{2}$. Then

$$
\begin{aligned}
& \int_{A_{6} \cap B_{1} \cap C_{2}} \prod_{j=1}^{3}\left|f\left(x_{j}-s_{j}\right)-f\left(x_{j}\right)\right|\left|K_{T}^{\theta}(s)\right| d s \\
& \leq C \int_{r / 2}^{\infty} \int_{s_{1} / 2}^{s_{1}-\delta} \int_{s_{1}-s_{2}-1 / T}^{s_{1}-s_{2}} s_{1}^{-2}\left(s_{1}-s_{2}\right)^{-1} \\
& \cdot \prod_{j=1}^{3}\left|f\left(x_{j}-s_{j}\right)\right| d s \leq C \sum_{i=0}^{\infty}(i \vee 1)^{-3 / 2} \\
& \cdot \sum_{0 \leq j<(i+1) / 2}(j \vee 1)^{-3 / 2} \\
& \cdot \int_{i}^{i+1} \int_{s_{1}-j-1}^{s_{1}-j} \int_{s_{1}-s_{2}-1 / T}^{s_{1}-s_{2}}\left|f\left(x_{1}-s_{1}\right)\right|\left|f\left(x_{2}-s_{2}\right)\right| \\
& \cdot\left|f\left(x_{3}-s_{3}\right)\right| d s_{1} d s_{2} d s_{3} \leq C \| f \times f \\
& \times f 1_{\left\{s: 0<s_{1}<N_{0}, s_{1} / 2<s_{2} \leq s_{1}-\delta, s_{1}-s_{2}-\delta<s_{3}<s_{1}-s_{2}\right\}} \|_{W\left(L_{1}, \ell_{\infty}\right)} \\
& +C N_{0}^{-1 / 2}\|f \times f \times f\|_{W\left(L_{1}, \ell_{\infty}\right)},
\end{aligned}
$$

which is small enough if $N_{0}$ is large and $\delta$ is small enough. On $\mathrm{A}_{6} \cap B_{1} \cap C_{3}$ we use the estimation

$$
\left|K_{T}^{\theta}(s)\right| \leq C s_{1}^{-1} s_{2}^{-1} s_{3}^{-1} \leq C s_{1}^{-2} s_{2}^{-1}
$$

to obtain

$$
\begin{aligned}
& \int_{A_{6} \cap B_{1} \cap C_{3}} \prod_{j=1}^{3}\left|f\left(x_{j}-s_{j}\right)-f\left(x_{j}\right)\right|\left|K_{T}^{\theta}(s)\right| d s \\
& \quad \leq C \int_{r / 2}^{\infty} \int_{\delta}^{s_{1} / 2} \int_{s_{1}-s_{2}-1 / T}^{s_{1}-s_{2}} s_{1}^{-2} s_{2}^{-1} \prod_{j=1}^{3}\left|f\left(x_{j}-s_{j}\right)\right| d s \\
& \leq C \| f \times f \\
& \quad \times f 1_{\left\{s: 0<s_{1}<N_{0}, \delta<s_{2} \leq s_{1} / 2, s_{1}-s_{2}-\delta<s_{3}<s_{1}-s_{2}\right\}} \|_{W\left(L_{1}, \ell_{\infty}\right)} \\
& +C N_{0}^{-1 / 2}\|f \times f \times f\|_{W\left(L_{1}, \ell_{\infty}\right)}<\epsilon,
\end{aligned}
$$

as just before.

On $A_{6} \cap B_{2} s_{1}-s_{2}>\delta$ again. If $s_{1}-s_{2}>2 \delta$, then $s_{3}>s_{1}-$ $s_{2}-\delta>\left(s_{1}-s_{2}\right) / 2$ and if $s_{1}-s_{2}<2 \delta$, then $s_{3}>\delta>\left(s_{1}-s_{2}\right) / 2$. This case can be handled in the same way as the set $A_{6} \cap B_{1}$.

On $A_{6} \cap B_{3}$ we get

$$
\begin{aligned}
\left|K_{T}^{\theta}(s)\right| & \leq C T^{-\alpha} s_{1}^{-1} s_{2}^{-1} s_{3}^{-1}\left(s_{1}-s_{2}-s_{3}\right)^{-\alpha} \\
& \leq C T^{-\alpha} s_{1}^{-1} s_{2}^{-1}\left(s_{1}-s_{2}\right)^{-1}\left(s_{1}-s_{2}-s_{3}\right)^{-\alpha}
\end{aligned}
$$

which implies that

$$
\begin{aligned}
& \int_{A_{6} \cap B_{3} \cap C_{2}} \prod_{j=1}^{3}\left|f\left(x_{j}-s_{j}\right)-f\left(x_{j}\right)\right|\left|K_{T}^{\theta}(s)\right| d s \\
& \leq C T^{-\alpha} \int_{r / 2}^{\infty} \int_{s_{1} / 2}^{s_{1}-\delta} \int_{\left(s_{1}-s_{2}\right) / 2}^{s_{1}-s_{2}-\delta} s_{1}^{-2}\left(s_{1}-s_{2}\right)^{-1} \\
& \cdot\left(s_{1}-s_{2}-s_{3}\right)^{-\alpha} \prod_{j=1}^{3}\left|f\left(x_{j}-s_{j}\right)\right| d s \\
& \leq C T^{-\alpha} \sum_{i=0}^{\infty}(i \vee 1)^{-1-\mu} \sum_{0 \leq j<(i+1) / 2}(j \vee 1)^{-1-\mu} \\
& \cdot \sum_{0 \leq k<(j+1) / 2}(k \vee 1)^{-1-\alpha+2 \mu} \\
& \cdot \int_{i}^{i+1} \int_{s_{1}-j-1}^{s_{1}-j} \int_{s_{1}-s_{2}-k-1}^{s_{1}-s_{2}-k}\left|f\left(x_{1}-s_{1}\right)\right|\left|f\left(x_{2}-s_{2}\right)\right| \\
& \cdot\left|f\left(x_{3}-s_{3}\right)\right| d s_{1} d s_{2} d s_{3} \\
& \leq C T^{-\alpha}\|f\|_{W\left(L_{1}, \ell_{\infty}\right)}^{3} \longrightarrow 0,
\end{aligned}
$$

where $0<\mu<1 / 2 \wedge \alpha / 2$. Similarly,

$$
\begin{aligned}
& \int_{A_{6} \cap B_{3} \cap C_{3}} \prod_{j=1}^{3}\left|f\left(x_{j}-s_{j}\right)-f\left(x_{j}\right)\right|\left|K_{T}^{\theta}(s)\right| d s \\
& \leq C T^{-\alpha} \int_{r / 2}^{\infty} \int_{\delta}^{s_{1} / 2} \int_{\left(s_{1}-s_{2}\right) / 2}^{s_{1}-s_{2}-\delta} s_{1}^{-2} s_{2}^{-1}\left(s_{1}-s_{2}-s_{3}\right)^{-\alpha} \\
& \cdot \prod_{j=1}^{3}\left|f\left(x_{j}-s_{j}\right)\right| d s \leq C T^{-\alpha} \sum_{i=0}^{\infty}(i \vee 1)^{-1-\mu} \\
& \cdot \sum_{0 \leq j<(i+1) / 2}(j \vee 1)^{-1-\mu} \sum_{0 \leq k<(j+1) / 2}(k \vee 1)^{-1-\alpha+2 \mu} \\
& \cdot \int_{i}^{i+1} \int_{j}^{j+1} \int_{s_{1}-s_{2}-k-1}^{s_{1}-s_{2}-k}\left|f\left(x_{1}-s_{1}\right)\right|\left|f\left(x_{2}-s_{2}\right)\right| \\
& \cdot\left|f\left(x_{3}-s_{3}\right)\right| d s_{1} d s_{2} d s_{3} \\
& \leq C T^{-\alpha}\|f\|_{W\left(L_{1}, \ell_{\infty}\right)}^{3} \longrightarrow 0 .
\end{aligned}
$$

Moreover, on $A_{6} \cap B_{4}$

$$
\begin{gathered}
\left|K_{T}^{\theta}(s)\right| \leq C T^{-\alpha} s_{1}^{-1} s_{2}^{-1} s_{3}^{-1}\left(s_{1}-s_{2}\right)^{-\alpha}, \\
\int_{A_{6} \cap B_{4} \cap C_{2}} \prod_{j=1}^{3}\left|f\left(x_{j}-s_{j}\right)-f\left(x_{j}\right)\right|\left|K_{T}^{\theta}(s)\right| d s \\
\leq C T^{-\alpha} \int_{r / 2}^{\infty} \int_{s_{1} / 2}^{s_{1}-\delta} \int_{\delta}^{\left(s_{1}-s_{2}\right) / 2} s_{1}^{-2}\left(s_{1}-s_{2}\right)^{-\alpha} \\
\cdot s_{3}^{-1} \prod_{j=1}^{3}\left|f\left(x_{j}-s_{j}\right)\right| d s \leq C T^{-\alpha} \sum_{i=0}^{\infty}(i \vee 1)^{-1-\mu}
\end{gathered}
$$




$$
\begin{aligned}
& \cdot \sum_{0 \leq j<(i+1) / 2}(j \vee 1)^{-1-\alpha+2 \mu} \sum_{0 \leq k<(j+1) / 2}(k \vee 1)^{-1-\mu} \\
& \cdot \int_{i}^{i+1} \int_{s_{1}-j-1}^{s_{1}-j} \int_{k}^{k+1}\left|f\left(x_{1}-s_{1}\right)\right|\left|f\left(x_{2}-s_{2}\right)\right| \\
& \cdot\left|f\left(x_{3}-s_{3}\right)\right| d s_{1} d s_{2} d s_{3} \leq C T^{-\alpha}\|f\|_{W\left(L_{1}, \ell_{\infty}\right)}^{3} \\
& \longrightarrow 0, \\
& \int_{A_{6} \cap B_{4} \cap C_{3}} \prod_{j=1}^{3}\left|f\left(x_{j}-s_{j}\right)-f\left(x_{j}\right)\right|\left|K_{T}^{\theta}(s)\right| d s \\
& \leq C T^{-\alpha} \int_{r / 2}^{\infty} \int_{\delta}^{s_{1} / 2} \int_{\delta}^{\left(s_{1}-s_{2}\right) / 2} s_{1}^{-1-\alpha} s_{2}^{-1} s_{3}^{-1} \prod_{j=1}^{3}\left|f\left(x_{j}-s_{j}\right)\right| d s \\
& \leq C T^{-\alpha} \sum_{i=0}^{\infty}(i \vee 1)^{-1-\alpha / 2} \sum_{0 \leq j<(i+1) / 2}(j \vee 1)^{-1-\alpha / 2} \\
& \quad \sum_{0 \leq k<(j+1) / 2}(k \vee 1)^{-1-\alpha / 2} \int_{i}^{i+1} \int_{j}^{j+1} \int_{k}^{k+1}\left|f\left(x_{1}-s_{1}\right)\right| \\
& \cdot\left|f\left(x_{2}-s_{2}\right)\right|\left|f\left(x_{3}-s_{3}\right)\right| d s_{1} d s_{2} d s_{3} \\
& \leq C T^{-\alpha}\|f\|_{W\left(L_{1}, \ell_{\infty}\right)}^{3} \longrightarrow 0 .
\end{aligned}
$$

Since $A_{6} \cap B_{5}=\emptyset$, the proof of the theorem is complete.

Corollary 6. Suppose that (14) and (25) hold for some even $d \in \mathbb{N}$ and $0<\alpha<\infty$. Let $f \in W\left(L_{1}, \ell_{q}\right)(\mathbb{R})$ for some $1 \leq q<\infty$. If $x \in \mathbb{R}$ is a Lebesgue point of $f$ and $f$ is locally bounded at $x$, then

$$
\lim _{T \rightarrow \infty} \frac{-1}{T} \int_{0}^{\infty} \theta^{\prime}\left(\frac{t}{T}\right)\left|s_{t} f(x)-f(x)\right|^{d} d t=0 .
$$

If $f$ is almost everywhere locally bounded, then the corollary holds almost everywhere. Unfortunately, it is not true that an integrable function is almost everywhere locally bounded (see [21]). The strong summability can be extended to all exponents in the usual way (see [21]).

Corollary 7. Suppose that (14) holds for all $d \in \mathbb{N}, \theta$ is nonincreasing, and $r>0$. Under the same conditions as in Corollaries 4 or 6, respectively, one gets that

$$
\lim _{T \rightarrow \infty} \frac{-1}{T} \int_{0}^{\infty} \theta^{\prime}\left(\frac{t}{T}\right)\left|s_{t} f(x)-f(x)\right|^{r} d t=0 .
$$

Note that under the same conditions we get for the Fejér summation that

$$
\lim _{T \rightarrow \infty} \frac{1}{T} \int_{0}^{T}\left|s_{t} f(x)-f(x)\right|^{r} d t=0 .
$$

\section{Strong Summability of Fourier Series}

In this section we formulate the above results for Fourier series. For an integrable function $f$ the $k$ th Fourier coefficient is defined by

$$
\widehat{f}(k)=\frac{1}{2 \pi} \int_{\mathbb{T}} f(x) e^{-\imath k x} d x \quad(k \in \mathbb{Z}),
$$

where $\mathbb{d}$ denotes the torus. For $f \in L_{1}(\mathbb{})$ and $n \in \mathbb{N}$ the $n$th partial sum $s_{n} f$ is introduced by

$$
\begin{aligned}
s_{n} f(x) & :=\sum_{k=-n}^{n} \widehat{f}(k) e^{\imath k x} \\
& =\frac{1}{2 \pi} \int_{\mathbb{T}} f(x-u) D_{n}(u) d u,
\end{aligned}
$$

where

$$
D_{n}(u):=\sum_{k=-n}^{n} e^{\imath k \cdot u}=2 \frac{\sin (n+1 / 2) x}{\sin (x / 2)} \quad(n \in \mathbb{N})
$$

is the Dirichlet kernel. Let

$$
\theta=\left(\theta(k, n), k \in \mathbb{N}, n \in \mathbb{N}_{+}\right)
$$

be a two-parameter sequence of real numbers satisfying

$$
\begin{aligned}
\theta(0, n) & =1, \\
\lim _{n \rightarrow \infty} \theta(k, n) & =1, \\
\lim _{k \rightarrow \infty} k^{d} \theta(k, n) & =0,
\end{aligned}
$$

$$
\sum_{k=0}^{\infty}(k+1)^{\beta}\left|\Delta_{1} \theta(k, n)\right| \leq C n^{\beta} \quad \text { for } \beta=0, d,
$$

where

$$
\Delta_{1} \theta(k, n):=\theta(k, n)-\theta(k+1, n) .
$$

The $\theta$-means of $f \in L_{1}(\mathbb{T})$ are defined by

$$
\begin{aligned}
\sigma_{n}^{\theta} f(x) & :=\sum_{k=-\infty}^{\infty} \theta(|k|, n) \widehat{f}(k) e^{\imath k x} \\
& =\frac{1}{2 \pi} \int_{\mathbb{T}} f(x-u) K_{n}^{\theta}(u) d u,
\end{aligned}
$$

where the $\theta$-kernel is given by

$$
K_{n}^{\theta}(x)=\sum_{j=0}^{\infty} \Delta_{1} \theta(j, n) D_{j}(x) .
$$

Hence

$$
\sigma_{n}^{\theta} f(x)=\sum_{j=0}^{\infty} \Delta_{1} \theta(j, n) s_{j} f(x) .
$$

Basically, we will consider two types of examples. The first type of examples is the Cesàro summation, which is generated by a sequence $\theta$.

Example 8 (Cesàro summation). For $k \in \mathbb{N}, \alpha \neq-1,-2, \ldots$, let

$$
\begin{aligned}
A_{k}^{\alpha} & :=\left(\begin{array}{c}
k+\alpha \\
k
\end{array}\right)=\frac{(\alpha+1)(\alpha+2) \cdots(\alpha+k)}{k !} \\
& =O\left(k^{\alpha}\right) \quad(k \in \mathbb{N})
\end{aligned}
$$


(see Zygmund [26, p. 77]). Let

$$
\theta(k, n)= \begin{cases}\frac{A_{n-k-1}^{\alpha}}{A_{n-1}^{\alpha}} & \text { if }|k| \leq n-1 \\ 0 & \text { if }|k| \geq n\end{cases}
$$

for some $0<\alpha<\infty$. Since $\Delta_{1} \theta(k, n)=A_{n-k-1}^{\alpha-1} / A_{n-1}^{\alpha}$, the Cesàro operators can be given by

$$
\sigma_{n}^{\theta} f(x)=\frac{1}{A_{n-1}^{\alpha}} \sum_{k=0}^{n-1} A_{n-1-k}^{\alpha-1} s_{k} f(x) .
$$

If $\alpha=1$, we get back the Fejér means.

The other type is generated by a function $\theta: \mathbb{R}_{+} \rightarrow \mathbb{R}$ (see all other examples in this paper). Let $\theta$ be continuous on $\mathbb{R}_{+}$and

$$
\theta(k, n):=\theta\left(\frac{k}{n}\right) \quad\left(k \in \mathbb{N}, n \in \mathbb{N}_{+}\right)
$$

Suppose that

$$
\begin{aligned}
& \theta(0)=1, \\
& \lim _{t \rightarrow \infty} t^{d} \theta(t)=0, \\
& \sum_{k=0}^{\infty}(k+1)^{\beta}\left|\Delta_{1} \theta\left(\frac{k}{n}\right)\right| \leq C n^{\beta} \quad \text { for } \beta=0, d .
\end{aligned}
$$

Note that for the Fejér means we get the usual definition

$$
\sigma_{n}^{\theta} f(x)=\frac{1}{n} \sum_{j=0}^{n-1} s_{j} f(x) .
$$

All examples of this paper satisfy (26) and (27).

Theorem 9. Suppose that (26), (27), and (69) are satisfied for some $d \in \mathbb{N}$ and $0<\alpha<\infty$. Let $f \in L_{p}(\mathbb{T})$ for some $1<p<$ $\infty$. If $x_{j}$ is a $p$-Lebesgue point of $f$ for all $j=1, \ldots, d$, then

$$
\lim _{n \rightarrow \infty} \sum_{k=0}^{\infty} \Delta_{1} \theta(k, n) \prod_{j=1}^{d}\left(s_{k} f\left(x_{j}\right)-f\left(x_{j}\right)\right)=0 .
$$

Moreover, if $d$ is even, then

$$
\lim _{n \rightarrow \infty} \sum_{k=0}^{\infty} \Delta_{1} \theta(k, n)\left|s_{k} f\left(x_{1}\right)-f\left(x_{1}\right)\right|^{d}=0 .
$$

Theorem 10. Suppose that (26), (27), and (69) are satisfied for some $d \in \mathbb{N}$ and $0<\alpha<\infty$. If $f \in L_{1}(\mathbb{T}), x_{j}$ is a Lebesgue point of $f$, and $f$ is locally bounded at $x_{j}$ for all $j=1, \ldots, d$, then (80) and (81) hold.

Corollary 11. Suppose that $r>0, \Delta_{1} \theta(k, n) \leq 0$, and (69) is satisfied for all $d$. Under the same conditions as in Theorems 9 or 10 , respectively, one gets that

$$
\lim _{n \rightarrow \infty} \sum_{k=0}^{\infty} \Delta_{1} \theta(k, n)\left|s_{k} f(x)-f(x)\right|^{r}=0 .
$$

Note that under the same conditions we get for the Fejér summation that

$$
\lim _{n \rightarrow \infty} \frac{1}{n} \sum_{k=0}^{n-1}\left|s_{k} f(x)-f(x)\right|^{r}=0 .
$$

Finally we note that the Weierstrass, Abel, Picard, Bessel, Fejér, de La Vallée-Poussin, Rogosinski, and Riesz summations can be considered as special cases of $\theta$-summation (see Weisz [21]).

\section{Conflict of Interests}

The author declares that there is no conflict of interests regarding the publication of this paper.

\section{Acknowledgments}

The author would like to thank the referee for reading the paper carefully and for calling his attention to some papers of the references. This research was supported by the Hungarian Scientific Research Funds (OTKA) no. K115804.

\section{References}

[1] H. Lebesgue, "Recherches sur la convergence des séries de Fourier," Mathematische Annalen, vol. 61, no. 2, pp. 251-280, 1905.

[2] L. Fejér, "Untersuchungen über Fouriersche Reihen," Mathematische Annalen, vol. 58, no. 1-2, pp. 51-69, 1903.

[3] G. H. Hardy and J. E. Littlewood, "Sur la séries de Fourier d'une fonction a carré sommable," Comptes Rendus (Paris), vol. 56, pp. 1307-1309, 1913.

[4] D. V. Giang and F. Móricz, "The strong summability of Fourier transforms," Acta Mathematica Hungarica, vol. 65, no. 4, pp. 403-419, 1994.

[5] G. H. Hardy and J. E. Littlewood, "The strong summability of Fourier series," Fundamenta Mathematicae, vol. 25, pp. 162-189, 1935.

[6] J. Marcinkiewicz, "Sur la sommabilité forte des series de Fourier," Journal of the London Mathematical Society, vol. 14, pp. $162-168,1939$.

[7] A. Zygmund, "On the convergence and summability of power series on the circle II," Proceedings of the London Mathematical Society, vol. 47, pp. 326-350, 1941.

[8] N. K. Bary, A Treatise on Trigonometric Series, Pergamon Press, Oxford, UK, 1964.

[9] O. D. Gabisoniya, "On the points of strong summability of Fourier series," Matematicheskie Zametki, vol. 14, pp. 615-626, 1973, Translation in Mathematical Notes, vol. 14, pp. 913-918, 1973 .

[10] E. S. Belinsky, "Summability of Fourier series with the method of lacunary arithmetical means at the Lebesgue points," Proceedings of the American Mathematical Society, vol. 125, no. 12, pp. 3689-3693, 1997.

[11] E. S. Belinsky, "Strong summability for the Marcinkiewicz means in the integral metric and related questions," Australian Mathematical Society, Series A: Pure Mathematics and Statistics, vol. 65, no. 3, pp. 303-312, 1998. 
[12] E. S. Belinsky, "Summability of multiple Fourier series," in Lebesgue Points: Theory of Functions, Functional Analysis and Their Applications, vol. 23, pp. 3-12, 1975, (Russian).

[13] E. S. Belinsky, E. R. Liyand, and R. M. Trigub, "The Banach algebra $\mathrm{A}^{*}$ and its properties," Journal of Fourier Analysis And Applications, vol. 3, pp. 103-129, 1997.

[14] P. L. Butzer and R. J. Nessel, Fourier Analysis and Approximation, Birkhauser, Basel, Switzerland, 1971.

[15] E. S. Belinsky, "Application of the Fourier transform to summability of Fourier series," Sibirskiu Matematicheskiu Zhurnal, vol. 18, pp. 497-511, 1977 (Russian), Translated in English: Siberian Mathematical Journal, vol. 18, pp. 353-363, 1977.

[16] R. M. Trigub, "Linear summation methods and absolute convergence of Fourier series," Izvestiya Akademii Nauk SSSR. Seriya Matematicheskaya, vol. 32, pp. 24-49, 1968.

[17] R. M. Trigub and E. S. Belinsky, Fourier Analysis and Approximation of Functions, Kluwer Academic Publishers, Dordrecht, The Netherlands, 2004.

[18] E. Liflyand, "Lebesgue constants of multiple Fourier series," Online Journal of Analytic Combinatorics, vol. 1, article 5, 112 pages, 2006.

[19] F. Weisz, Summability of Multi-Dimensional Fourier series and Hardy Spaces, vol. 541 of Mathematics and its Applications, Kluwer Academic Publishers, Dordrecht, The Netherlands, 2002.

[20] F. Weisz, "Summability of multi-dimensional trigonometric fourier series," Surveys in Approximation Theory, vol. 7, pp. 1179, 2012.

[21] F. Weisz, "Multi-dimensional Fourier transforms, Lebesgue points and strong summability," Mediterranean Journal of Mathematics, In press.

[22] H. G. Feichtinger and F. Weisz, "The Segal algebra $S_{0}\left(\mathbb{R}^{d}\right)$ and norm summability of Fourier series and Fourier transforms," Monatshefte für Mathematik, vol. 148, pp. 333-349, 2006.

[23] H. G. Feichtinger and F. Weisz, "Wiener amalgams and pointwise summability of FOUrier transforms and FOUrier series," Mathematical Proceedings of the Cambridge Philosophical Society, vol. 140, no. 3, pp. 509-536, 2006.

[24] L. Grafakos, Classical and Modern Fourier Analysis, Pearson Education, Upper Saddle River, NJ, USA, 2004.

[25] F. Weisz, "Marcinkiewicz-summability of multi-dimensional Fourier transforms and Fourier series," Journal of Mathematical Analysis and Applications, vol. 379, no. 2, pp. 910-929, 2011.

[26] A. Zygmund, Trigonometric Series, Cambridge University Press, London, UK, 3rd edition, 2002. 


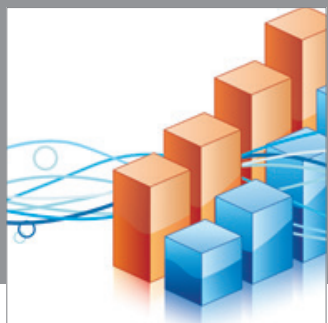

Advances in

Operations Research

mansans

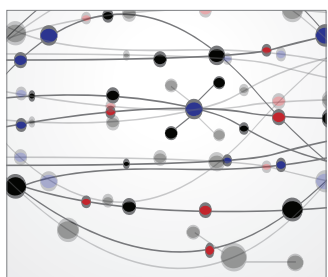

The Scientific World Journal
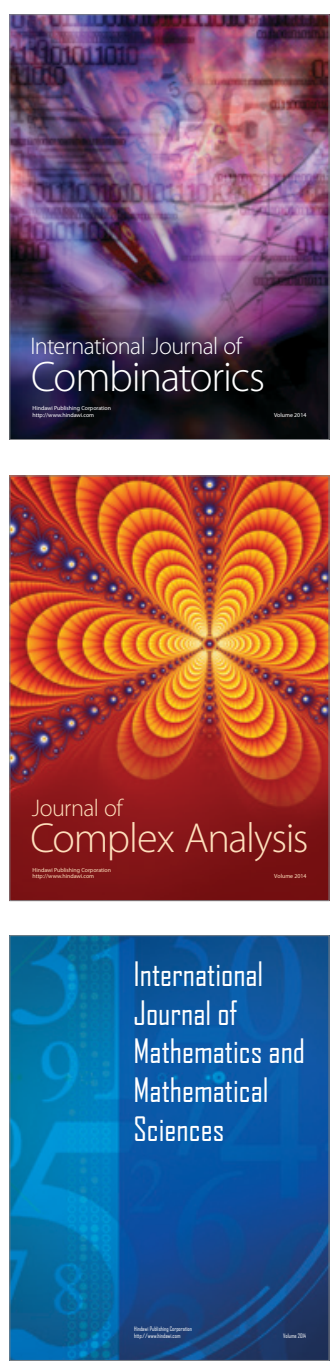
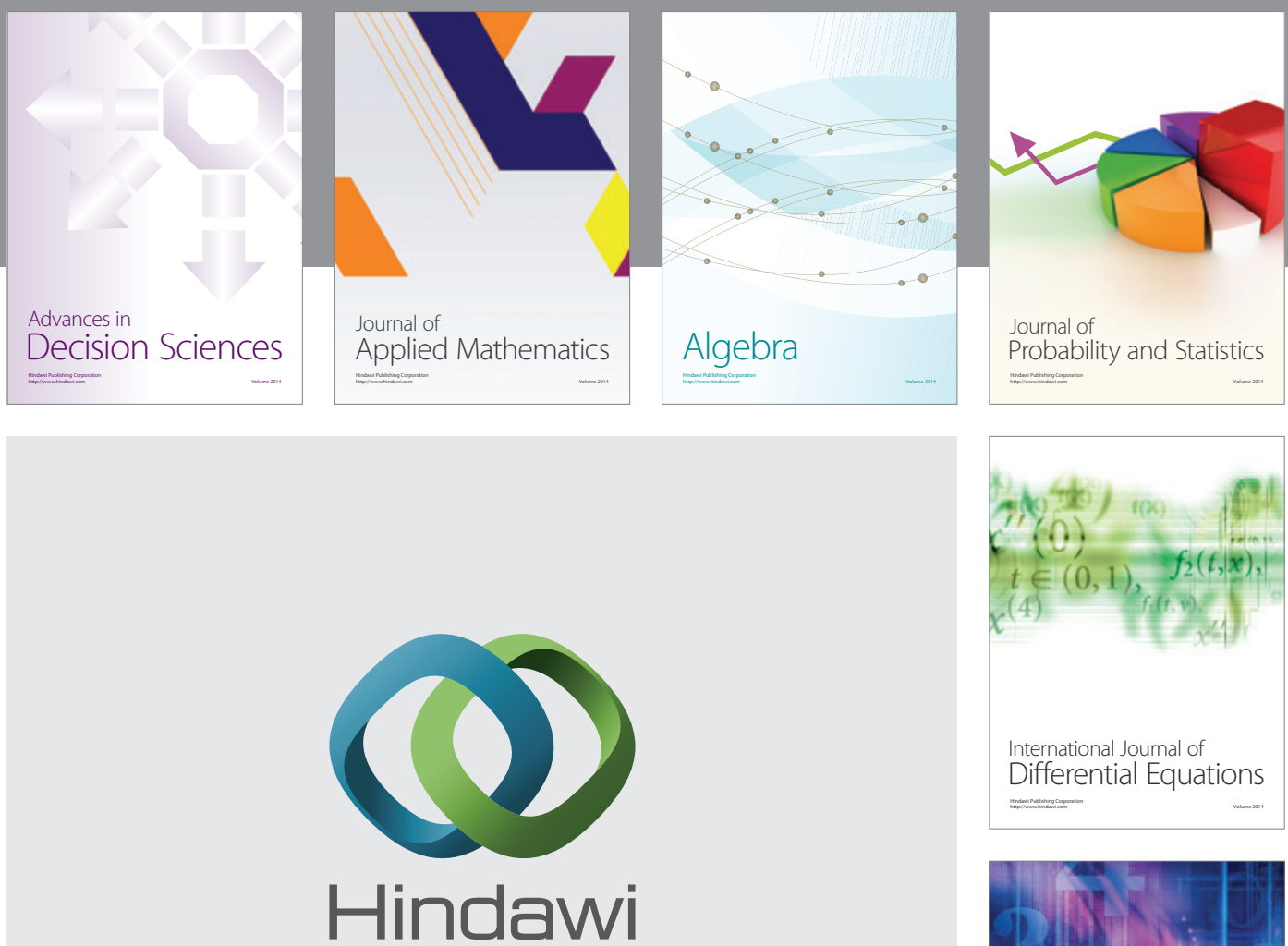

Submit your manuscripts at http://www.hindawi.com
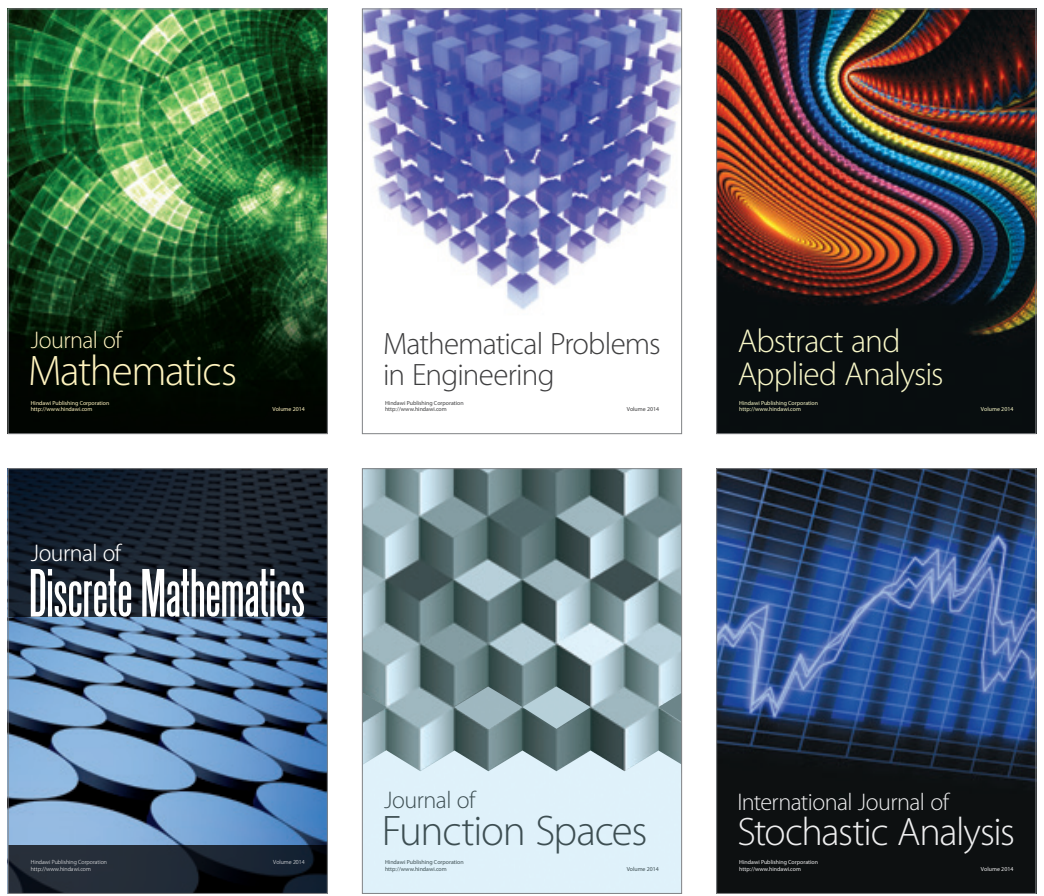

Journal of

Function Spaces

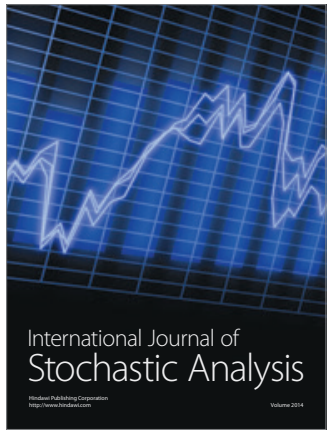

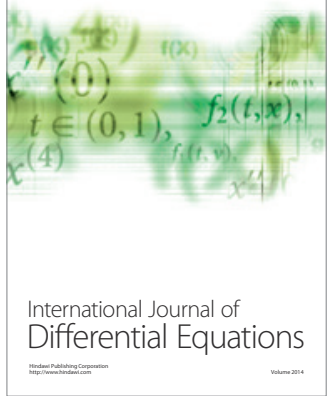
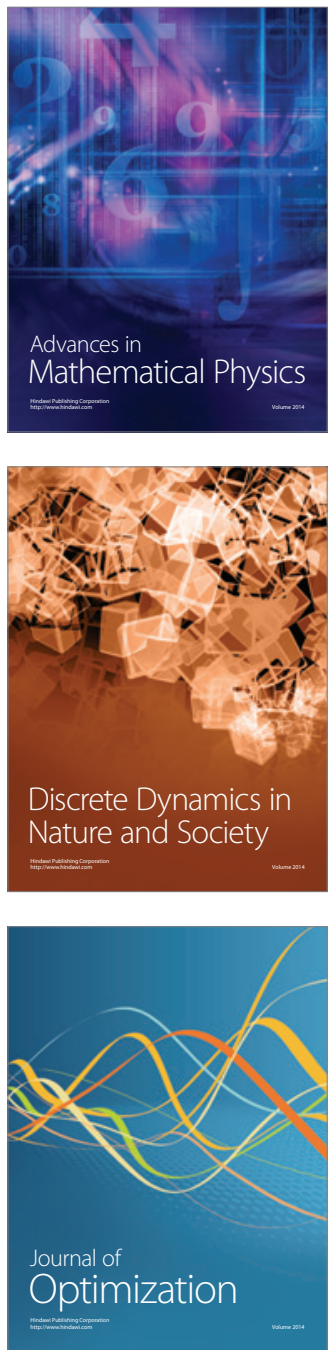